\title{
CHARACTERIZATIONS FOR THE FRACTIONAL INTEGRAL OPERATOR AND ITS COMMUTATORS IN GENERALIZED WEIGHTED MORREY SPACES ON CARNOT GROUPS
}

\author{
VAGIF S. GULIYEV* AND ISMAIL EKINCIOGLU
}

\begin{abstract}
In this paper, we shall give a characterization for the strong and weak type Spanne type boundedness of the fractional integral operator $I_{\alpha}, 0<\alpha<Q$ on Carnot group $\mathbb{G}$ on generalized weighted Morrey spaces $M_{p, \varphi}(\mathbb{G}, w)$, respectively, where $Q$ is the homogeneous dimension of $\mathbb{G}$. Also we give a characterization for the Spanne type boundedness of the commutator operator $\left[b, I_{\alpha}\right]$ on generalized weighted Morrey spaces.

As applications of the properties of the fundamental solution of sub-Laplacian $\mathscr{L}$ on $\mathbb{G}$, we prove two Sobolev-Stein embedding theorems on generalized weighted Morrey spaces in the Carnot group setting.
\end{abstract}

Mathematics subject classification (2010): Primary 42B25, 42B35, 43A15, 43A80.

Keywords and phrases: Carnot group, fractional integral operator, generalized weighted Morrey space, commutator, $B M O$.

\section{REFERENCES}

[1] A. Bonfiglioli, E. Lanconelli, F. Uguzzoni, Stratified Lie groups and potential theory for their sub-Laplacians, Springer Monographs in Mathematics. Springer, Berlin (2007).

[2] A. Bernardis, S. Hartzstein, G. Pradolini, Weighted inequalities for commutators of fractional integrals on spaces of homogeneous type, J. Math. Anal. Appl. 322, 825-846 (2006).

[3] G. B. Folland, A fundamental solution for a subelliptic operator, Bull. Amer. Math. Soc. 79 (2), 373-376 (1973).

[4] G. B. Folland, Subelliptic estimates and function spaces on nilpotent Lie groups, Ark. Mat. 13, 161-207 (1975).

[5] G. B. Folland, E. M. Stein, Hardy Spaces on homogeneous Groups, Mathematical Notes Vol. 28, Princeton Univ. Press, Princeton (1982).

[6] G. Furioli, C. Melzi, A. Veneruso, Littlewood-Paley decompositions and Besov spaces on Lie groups of polynomial growth, Math. Nachr. 279 (9-10), 1028-1040 (2006).

[7] F. DERINGOZ, Parametric Marcinkiewicz integral operator and its higher order commutators on generalized weighted Morrey spaces, Trans. Natl. Acad. Sci. Azerb. Ser. Phys.-Tech. Math. Sci. 37 (4), Mathematics, 24-32 (2017).

[8] F. Deringoz, V. S. Guliyev, S. G. Hasanov, Maximal operator and its commutators on generalized weighted Orlicz-Morrey spaces, Tokyo J. Math. 41 (2), 347-369 (2018).

[9] A. Eroglu, V. S. Guliyev, C. V. AzIzov, Characterizations for the fractional integral operators in generalized Morrey spaces on Carnot groups, Math. Notes 102 (5-6), 722-734 (2017).

[10] A. ERoglu, C. V. AzIZov, A note of the fractional integral operators in generalized Morrey spaces on the Heisenberg group, Trans. Natl. Acad. Sci. Azerb. Ser. Phys.-Tech. Math. Sci. 37 (1), Mathematics, 86-91 (2017).

[11] S. Giulini, Approximation and Besov spaces on stratified groups, Proc. Amer. Math. Soc. 96 (4), 569-578 (1986).

[12] V. S. GULIYEV, Integral operators on function spaces on the homogeneous groups and on domains in $\mathbb{G}$, (Russian) Doctor's degree dissertation, Moscow, Mat. Inst. Steklov, 1-329 (1994). 
[13] V. S. GuliYev, Function spaces, integral operators and two weighted inequalities on homogeneous groups. Some applications, (Russian) Baku, 1-332 (1999).

[14] V. S. Guliyev, Boundedness of the maximal, potential and singular operators in the generalized Morrey spaces, J. Inequal. Appl. Art. ID 503948, 20 pp. (2009).

[15] V. S. GuLIYEV, Generalized local Morrey spaces and fractional integral operators with rough kernel, J. Math. Sci. (N. Y.) 193 (2), 211-227 (2013).

[16] V. S. Guliyev, A. Eroglu, Y. Y. Mammadov, Riesz potential in generalized Morrey spaces on the Heisenberg group, Problems in mathematical analysis. No. 68. J. Math. Sci. (N. Y.) 189 (3), 365382 (2013).

[17] V. S. Guliyev, A. Akbulut, Y. Y. Mammadov, Boundedness of fractional maximal operator and their higher order commutators in generalized Morrey spaces on Carnot groups, Acta Math. Sci. Ser. B Engl. Ed. 33 (5), 1329-1346 (2013).

[18] V. S. GuliYeV, Generalized weighted Morrey spaces and higher order commutators of sublinear operators, Eurasian Math. J. 3 (3), 33-61 (2012).

[19] V. S. GuliYeV, M. N. OMAROVA, Multilinear singular and fractional integral operators on generalized weighted Morrey spaces, Azerb. J. Math. 5 (1), 104-132 (2015).

[20] V. S. GuliYeV, V. H. HAMZAYEV, Rough singular integral operators and its commutators on generalized weighted Morrey spaces, Math. Ineq. Appl. 19 (3), 863-881 (2016).

[21] V. S. GuliYeV, J. J. HASAnOV, X. A. BADAlov, Maximal and singular integral operators and their commutators on generalized weighted Morrey spaces with variable exponent, Math. Inequal. Appl. 21 (1), 41-61 (2018).

[22] V. S. Guliyev, J. J. Hasanov, X. A. Badalov, Commutators of the potential type operators in the vanishing generalized weighted Morrey spaces with variable exponent, Math. Inequal. Appl. 22 (1), 331-351 (2019).

[23] V. S. Guliyev, I. Ekincioglu, E. Kaya, Z. Safarov, Characterizations for the fractional maximal operator and its commutators in generalized Morrey spaces on Carnot groups, Integral Transforms Spec. Funct. 30 (6), 453-470 (2019).

[24] V. H. HAMZAYEV, Sublinear operators with rough kernel generated by Calderon-Zygmund operators and their commutators on generalized weighted Morrey spaces, Trans. Natl. Acad. Sci. Azerb. Ser. Phys.-Tech. Math. Sci. 38 (1), Mathematics, 79-94 (2018).

[25] K.-P. Ho, Characterizations of BMO by $A_{p}$ weights and p-convexity, Hiroshima Math. J. 41, 153165 (2011).

[26] A. Kassymov, M. RuZhansk y, D. Suragan, Hardy-Littlewood-Sobolev and Stein-Weiss inequalities on homogeneous Lie groups, Integral Transforms Spec. Funct. 30 (8), 643-655 (2019).

[27] A. KaPLan, Fundamental solutions for a class of hypoelliptic PDE generated by composition of quadratics forms, Trans. Amer. Math. Soc. 258, 147-153 (1980).

[28] Y. KOMORI, S. ShIRAI, Weighted Morrey spaces and a singular integral operator, Math. Nachr. 282 (2), 219-231 (2009).

[29] B. Muckenhoupt, Weighted norm inequalities for the Hardy maximal function, Trans. Amer. Math. Soc. 165, 207-226 (1972).

[30] B. Muckenhoupt, R. Wheeden, Weighted norm inequalities for fractional integrals, Trans. Amer. Math. Soc. 192, 261-274 (1974).

[31] T. Mizuhara, Boundedness of some classical operators on generalized Morrey spaces, Harmonic Analysis (S. Igari, Editor), ICM 90 Satellite Proceedings, Springer-Verlag, Tokyo 183-189 (1991).

[32] C. B. Morrey, On the solutions of quasi-linear elliptic partial differential equations, Trans. Amer. Math. Soc. 43, 126-166 (1938).

[33] E. NAKAI, Hardy-Littlewood maximal operator, singular integral operators and Riesz potentials on generalized Morrey spaces, Math. Nachr. 166, 95-103 (1994).

[34] S. NAKAMURA, Generalized weighted Morrey spaces and classical operators, Math. Nachr. 289 (1718), 2235-2262 (2016).

[35] C. Perez, R. Wheeden, Uncertainty principle estimates for vector fields, J. Funct. Anal. 181, 146188 (2001).

[36] M. Ruzhansky, D. Suragan, N. Yessirkegenov, Hardy-Littlewood, Bessel-Riesz, and fractional integral operators in anisotropic Morrey and Campanato spaces, Fract. Calc. Appl. Anal. 21 (3), 577-612 (2018). 
[37] M. RuZhansky, D. Suragan, N. Yessirkegenov, Sobolev type inequalities, Euler-HilbertSobolev and spaces on homogeneous groups, Integral Equations Operator Theory 90 (1), Art. 10, 33 pp. (2018).

[38] K. SAKA, Besov spaces and Sobolev spaces on a nilpotent Lie group, Tohoku Math. J. 31, 383-437 (1979).

[39] L. SAloff-Coste, Inegalities de Sobolev produit sur les groupes de Lie nilpotents, J. Funct. Anal. 79, 44-56 (1988).

[40] Y. SAWAno, A thought on generalized Morrey spaces, J. Indonesian Math. Soc. 25 (3), 210-281 (2019).

[41] L. SKRZypCZAK, Besov spaces and Hausdorff dimension for some Carnot Caratheodory metric spaces, Canad. J. Math. 54, 1280-1304 (2002).

[42] E. M. STEIN, Harmonic analysis: Real-variable methods, orthogonality and oscillatory integrals, Princeton Univ. Press, Princeton (1993). 